Cahiers « Mondes anciens »

ANCIENS

Histoire et anthropologie des mondes anciens

$3 \mid 2012$

Femmes de paroles

\title{
Voix de Lacédémoniennes
}

Injonction et identité de genre dans les paroles de femmes spartiates

\section{Charles Delattre}

\section{(2) OpenEdition \\ Journals}

Édition électronique

URL : http://journals.openedition.org/mondesanciens/744

DOI : 10.4000/mondesanciens.744

ISSN : 2107-0199

Éditeur

UMR 8210 Anthropologie et Histoire des Mondes Antiques

Référence électronique

Charles Delattre, «Voix de Lacédémoniennes », Cahiers « Mondes anciens » [En ligne], 3 | 2012, mis en ligne le 23 mai 2012, consulté le 05 mai 2019. URL : http://journals.openedition.org/

mondesanciens/744; DOI : 10.4000/mondesanciens.744

Ce document a été généré automatiquement le 5 mai 2019.

\section{(c) (i) $\odot$}

Les Cahiers «Mondes Anciens " sont mis à disposition selon les termes de la licence Creative Commons Attribution - Pas d'Utilisation Commerciale - Pas de Modification 4.0 International. 


\title{
Voix de Lacédémoniennes
}

\author{
Injonction et identité de genre dans les paroles de femmes spartiates
}

\author{
Charles Delattre
}

1 Pour chercher la voix des femmes antiques, nous n'avons plus, c'est une évidence, qu'un matériau textuel qui les met singulièrement à distance, soit que nos documents ne reprennent tout simplement pas les paroles des femmes, soit qu'ils les transmettent en un discours sans cesse indirect, médiatisé par la voix d'un narrateur, transcripteur, auteur qui, même lorsqu'il semble s'effacer, reste néanmoins particulièrement opaque. La voix des femmes ne nous parvient a priori pas telle qu'elle s'est fait entendre, mais telle qu'on a bien voulu la représenter : même s'il peut y avoir coïncidence entre l'une et l'autre forme, le fait que la voix féminine est en représentation, et non en nature, doit nous inciter à la prudence.

\section{Apophtegmes au féminin}

2 On prendra donc ici pour sujet d'étude un type particulièrement narrativisé de parole, un énoncé qui est aussi une expression, l'apophtegme. Formulation brève, condensée, ramassée sur elle-même, l'apophtegme est à la fois un cri, un appel et une définition de soi, il est une voix qui dit la vérité de son énonciateur, auquel l'énonciateur - ou le narrateur qui construit un énonciateur - confie une vérité. On ne s'étonnera donc pas de trouver dans les ensembles textuels transmis dans les manuscrits sous le nom de Plutarque deux collections d'apophtegmes, puisque l'apopthegme, défini comme une expression prise sur le vif, réunit justement dans sa formulation deux des fondements de l'entreprise plutarquéenne, l'anecdote historique et l'èthos du personnage qui est au cœur de l'anecdote' ${ }^{1}$.

3 Le premier ensemble, Apophtegmes de rois et de généraux, est précédé d'une dédicace à Trajan et reproduit par ses divisions, des rois perses aux dirigeants romains, à la fois le cadre géographique et historique par lequel l'histoire d'époque hellénistique et romaine pensait le monde ${ }^{2}$. Le deuxième, Apophtegmes laconiens, est centré sur Sparte : il n'est plus

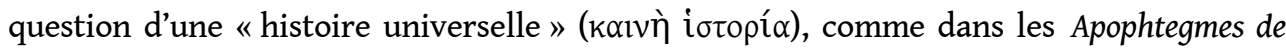
rois et de généraux, mais d'une histoire locale, celle de Sparte, qui a été figée en un 
«mythe » dès les $v^{e}$ et $\mathrm{IV}^{\mathrm{e}} \mathrm{s} .^{3}$, en particulier grâce à Platon et Xénophon, et qui s'est définitivement ossifiée à l'époque romaine.

4 Notons que le statut définitif de ces collections d'énoncés n'est pas forcément identique. Certes, l'un et l'autre texte sont vraisemblablement des documents de travail, le résultat de compilations diverses opérées par Plutarque lui-même et organisées pour servir de matériau aux Vies et aux Euvres morales. Mais les Apophtegmes des rois et des généraux semblent avoir fait l'objet d'une édition-publication (ekdosis) particulière de la part de Plutarque : le rapport entre ces Apophtegmes et la Vie de Lycurgue a été particulièrement étudié par W. NACHSTÄDT (1935, p.165-167), qui conclut à l'authenticité du matériel apophtegmatique et à la précédence de ce matériel sur la rédaction de la Vie. Il faut donc écarter l'hypothèse d'une mise en catalogue survenue dans un deuxième temps, à partir des œuvres de Plutarque ${ }^{4}$.

5 Les Apophtegmes laconiens peuvent donc être considérés comme un document de travail de Plutarque qui a atteint, dans les manuscrits médiévaux, le statut d'œuvre à part entière, même si telle n'était pas l'intention de leur auteur au départ. Ils s'insèrent d'ailleurs assez bien dans le catalogue plutarquéen, car ils participent eux-mêmes à leur façon à la reconduction du mirage spartiate, en contribuant à la diffusion du «mythe du laconisme »: les Spartiates s'exprimeraient préférentiellement sous une forme brève, toujours en réponse et jamais d'un premier mouvement, manifestant ainsi une maitrise de leur voix et de leur langage similaire à l'enkrateia générale qui serait la marque de l'éducation des Homoioi, telle que Plutarque l'a formalisée dans sa Vie de Lycurgue.

6 Surtout, les Apophtegmes laconiens contiennent explicitement des énoncés mis au compte de femmes et insérés dans une organisation plutôt lâche : 346 bons mots de dirigeants, dont les noms classés par ordre alphabétique organisent la liste, sont suivis de 72 apophtegmes anonymes; une troisième série est constituée par des remarques et des curiosités concernant des institutions, où l'énoncé formulaire a généralement disparu au profit d'une description; une dernière liste clôt le catalogue général, qui regroupe quarante apophtegmes attribués d'abord à quatre Laconiennes identifiées par leur nom (Archiléônis, Gorgô, Gyrtias, Damatria), puis à une série de Laconiennes anonymes.

7 Cette dernière partie, par son articulation entre personnages identifiés et anonymes, redouble les deux premières séries. Séparées des apophtegmes de dirigeants et de Spartiates anonymes par la troisième série, composée d'énoncés non apophtegmatiques, ces formules sont le versant féminin qui complète la parole des hommes spartiates mise en tête de catalogue ${ }^{5}$. Ces « voix de femmes " peuvent passer dans une première approche pour des énoncés rares, le témoignage d'une parole féminine où pourra justement se définir la part féminine de la parole, mais en fait rien ne les distingue formellement de la "voix des hommes» qui se fait entendre en début de catalogue: énoncés brutaux, formules brèves, teintées ici et là de dorien - sans qu'on puisse trouver cependant d'usage systématique, qui reproduirait la parole même d'un Spartiate -, remarques lapidaires ne dessinent pas les contours d'un genre particulier. L'injonction semble aussi bien masculine que féminine dans ce catalogue.

\section{La voix des mères}

8 Une polarité se dessine en revanche dans les thèmes abordés et dans la direction que prend l'injonction elle-même. La très grande majorité de ces Laconiennes sont en effet des mères et s'expriment en mettant en avant leur statut de mère: ou bien elles 
s'adressent directement à leur fils, ou bien leur parole porte sur leur fils ${ }^{6}$. De leur côté, les enfants de ces Laconiennes sont uniformément envisagés comme des soldats, et leur conduite est jugée à l'aune de l'exemplaire héroïsme spartiate que la collection d'apophtegmes vise à construire.

9 L'un de ces apophtegmes les plus célèbres - on le retrouve encore dans Astérix et le chaudron-est attribué à une Laconienne anonyme dans la collection de Plutarque et remonterait à Aristote, si l'on en croit Stobée ${ }^{7}$. Remettant son bouclier à son fils sur le point de partir au combat, la mère lui livre, comme souhait et comme admonition,

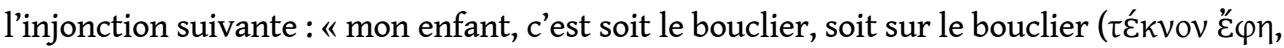

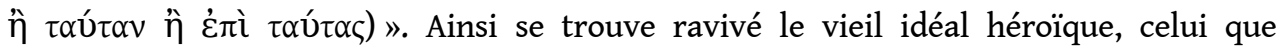
célébrait entre autres Tyrtée à Sparte même, qui incite le soldat à ne jamais se séparer de son bouclier, même au prix de sa vie ${ }^{8}$, et qui fait du bouclier plus qu'une arme, une partie même du corps'.

L'ordre de la mère s'oppose à une autre parole, celle, provocatrice, d'Archiloque, qui revendiquait au contraire sa survie au prix de l'abandon de ses armes ${ }^{10}$ :

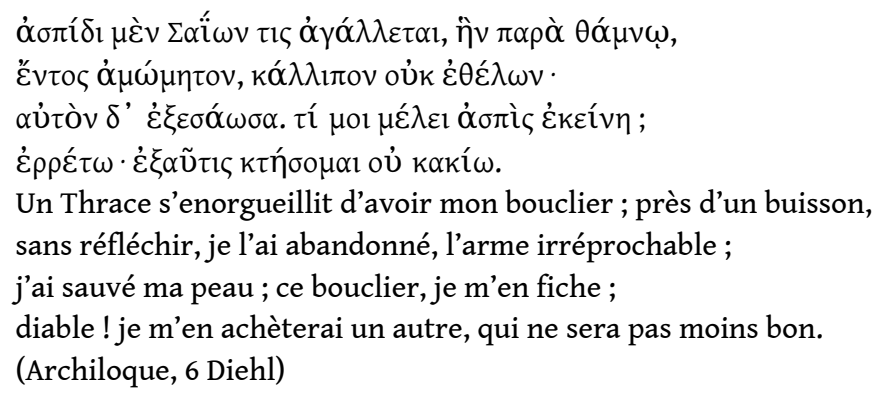

L'apophtegme de la mère laconienne s'appuie en même temps sur d'autres apophtegmes, qui font du corps même du soldat l'ultime défense, le rempart d'une ville et le bouclier par excellence, par exemple

une cité n'est pas sans défense, quand elle est couronnée d'hommes, et non de briques ${ }^{11}$.

Disjoindre le bouclier de ce corps, c'est anéantir le statut de soldat-citoyen, c'est revenir sur l'un des fondements de l'identité spartiate, qui unit le corps de ses soldats à la cité elle-même.

Les injonctions des mères ont pour fonction d'assigner à leur fils une place précise dans la société, une conduite et un èthos : elles sont précisément normatives, car elles définissent une identité, identité qui est elle-même exprimée sous la forme d'un modèle idéal auquel il convient de se soumettre. Le dialogue entre la mère et son fils n'en est pas un, il s'agit d'une parole à sens unique, d'où toute réponse est exclue : seules l'action, la vie et surtout la mort du fils peuvent servir de réponse à la parole maternelle.

Pour avoir plus de force, l'injonction de la mère Laconienne se fonde sur un paradoxe : mieux vaudrait avoir un fils mort qu'un fils qui ne respecterait pas cette injonction. On est bien loin de la mère que le chœur de l'Hécube d'Euripide décrit, une mère dont les fils sont morts et

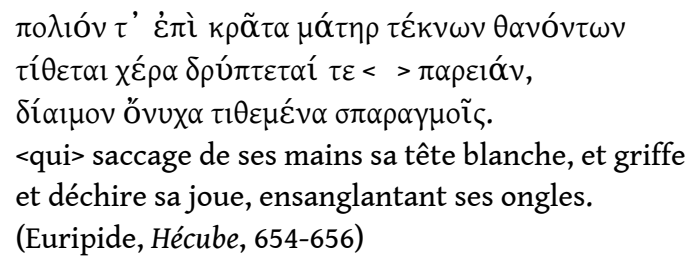




\section{su engendrer des enfants vigoureux ${ }^{12}$, comme celle qui a su les former en vrais Spartiates,} comme celle qui est capable de produire de tels énoncés. Contrairement à S. POMEROY (2002), je ne pense pas que ces énoncés permettent de reconstruire la société spartiate historique, ni même son idéologie ${ }^{13}$ : ces formules n'ont pas été construites par les Spartiates eux-mêmes, mais par ceux qui les admiraient. Elles sont le cœur même du «mirage spartiate» et jouent en écho avec d'autres éléments de ce mirage. Je serai d'autant moins enclin à conclure, à la suite de ces anecdotes, à l'existence d'une « maternité épanouie » (fulfilling motherhood) typiquement spartiate ${ }^{14}$.

Ce dont il s'agit, c'est de la construction de deux identités, partiellement fondées sur le genre, mais réorientées par la fonction sociale, celle de la mère laconienne et celle du guerrier spartiate. Chacune d'elles se construit sur une enkrateia absolue, qui vise à

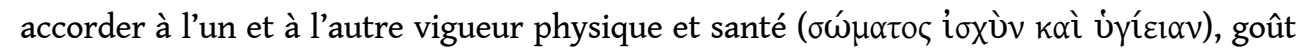

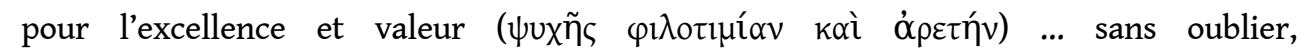
paradoxalement, un indispensable dédain pour l'opinion publique. L'idéologie spartiate ainsi construite s'oppose non seulement à l'opinion de ceux qui ne sont pas Spartiates, mais aussi à l'opinion des Spartiates qui n'ont pas su intégrer l'idéologie. On a donc la définition paradoxale d'une injonction qui définit un être social, en accord avec les exigences générales d'une société dont les membres ne promeuvent pas forcément cette même définition. Les apophtegmes ne dessinent pas une société spartiate uniforme, ni une idéologie unaniment partagée, mais au contraire une tension constante entre des Spartiates qui se conforment à un idéal ... et les autres, qui n'en sont pas moins Spartiates de droit. Raison pour laquelle il me semble difficile de transformer cette norme sociale en une réalité historique ${ }^{15}$.

17 En définitive, si les femmes et les enfants peuvent passer pour inférieurs à certains hommes ailleurs ${ }^{16}$, à Sparte ils se doivent d'être leurs égaux, ou devraient tenter de l'être. Est-ce parce que la femme est guerrière à sa façon, parce que la mère est au risque de mourir comme son fils, parce qu'elle est mère, parce qu'il est soldat, et ne renonce pas à courir ce risque $^{17}$ ? En d'autres termes, la mère spartiate, et non la femme, est l'égale du guerrier spartiate, et non de l'homme. La mère spartiate ne devrait pas être une femme comme les autres. Si elle est femme, ce critère n'est pas distinctif en soi, il ne fait que se combiner à d'autres ${ }^{18}$.

En conclusion, du point de vue des critères de genre, dans les énoncés apophtegmatiques, il y a discontinuité entre mère et femme : si Gorgô, épouse de Léonidas, peut affirmer à une Athénienne, dans un dialogue fictif, que les Laconiennes peuvent commander aux

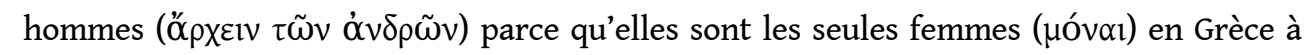
donner naissance à des hommes (ö $v \delta \rho \varepsilon \varsigma)^{19}$, il faut entendre cette formule ni comme le reflet des institutions spartiates historiques, ni comme un énoncé qui renvoie au genre, mais comme l'illustration par excellence de la formulation apophtegmatique : les mères spartiates peuvent délivrer des injonctions à leur fils, pour qu'ils se conduisent justement en hommes spartiates. S'ils n'obéissent pas à ces injonctions, le dernier ordre qu'elles leur adressent est celui de disparaitre, soit du territoire de Sparte, soit du monde des vivants ${ }^{20}$, à moins qu'elles n'exécutent elles-mêmes cette dernière menace ${ }^{21}$. L'ordre est d'abord ce qui passe par la parole, ici l'injonction, qui à son tour se transforme en apophtegme lorsqu'elle s'insère dans une liste et devient exemplaire. 


\section{Gorgô et l'injonction}

19 L'exemple de Gorgô est particulièrement intéressant, car cette femme est à la fois un personnage historique, l'épouse du roi Léonidas, et un personnage modèle que Plutarque insère aussi bien dans ses Apophtegmes laconiens que dans sa Vie de Lycurgue, et qu'il a trouvé chez Hérodote. Cette fille du roi Cléomène $\mathrm{I}^{\text {er }}$ occupe une position particulière dans la famille royale des Agiades : à la mort de son père, décédé apais, c'est-à-dire sans descendance mâle ${ }^{22}$, elle épouse non pas son oncle Dorieus, mort en rébellion, mais son oncle Léonidas. Quoiqu'elle ne transmette pas la royauté, elle semble constituer un élément de pouvoir et de légitimation. Elle apparait deux fois chez Hérodote, dans deux anecdotes complémentaires. Encore enfant, elle admoneste son père et lui enjoint de résister aux tentatives de corruption du tyran Aristagoras de Milet : devenu apophtegme, l'épisode se retrouve tel quel dans la liste de Plutarque ${ }^{23}$. Épouse de Léonidas, elle fait preuve non seulement de courage et d'enkrateia, mais aussi de perspicacité lorsque Démarate envoie un message secret aux Lacédémoniens pour les prévenir de l'invasion que le Grand Roi Xerxès projette : ayant reçu une tablette vierge, par l'intermédiaire d'un messager, les Spartiates ne savaient qu'en faire, jusqu'à ce que Gorgô leur suggère de gratter la cire, afin de découvrir le message qui avait été gravé dans le bois de la tablette, puis dissimulé24.

Dans ces deux anecdotes, Gorgô semble jouer le rôle de gardienne : surveillant son père, comme les Laconiennes le font pour leur fils, et assistant au désarroi des Homoioi, elle est d'abord un regard qui observe, qui fixe et qui voit tout. Je n'irai pas jusqu'à faire de Gorgô une nouvelle Gorgone, d'abord parce que son nom est bien attesté ailleurs et ne porte donc pas de charge particulière ${ }^{25}$; mais il y a peut-être quelque chose de l'ordre du jeu de mots ou d'intention dans la construction de ce personnage par Hérodote, présent là où on ne l'attend pas, vigilant et inquiet, qui redresse ce qui risquait de ployer, qui dénoue les fils d'un stratagème ( $\mu \eta \chi \alpha$ ó $v \eta \mu \alpha)$ subtil ${ }^{26}$. Gorgô réalise chez Hérodote l'union de l'intelligence rusée ( $\mu \tilde{\eta} \tau \imath \varsigma)$ et du regard scrutateur (үopүóc).

Gorgô n'est d'ailleurs pas que regard, mais voix aussi : c'est explicite dans l'anecdote qui l'oppose à son père, mais cela est visible aussi dans son intervention face aux Lacédémoniens. Elle ne prend pas la tablette elle-même, ne semble même pas vérifier son

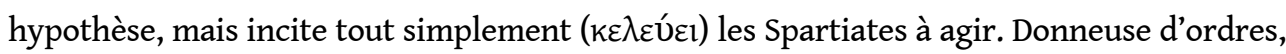
non parce qu'elle serait reine, mais parce qu'elle est fille de Spartiate, épouse de Spartiate et mère de Spartiate: la seule relation de paroles qu'elle puisse avoir avec eux, c'est l'injonction, qui rétablit l'ordre des choses. La voix ici redouble le coup d'œil, elle frappe au même titre que le regard, avec une efficacité certaine : de l'œil à la parole, une seule tension, une même émission, quasiment une seule et même action.

L'ordre spartiate, tel que le dessinent ces apophtegmes, c'est donc une parole injonctive ; c'est un modèle normatif qui unit la mère et son fils et qui renverse la posture attestée ailleurs de la mère pleurant son enfant mort à la guerre ; c'est la production d'un pouvoir institutionnel ( $\dot{\alpha} \rho \chi \eta ́)$ et la soumission à ce même pouvoir, sans discussion; c'est un modèle de gouvernement de soi et des autres, qui constitue l'eunomia, "l'ordre juste » spartiate. Pas d'isonomie ici, pas d'isègorie: chaque corps occupe une place et une fonction déterminées. Le Spartiate idéal, c'est celui qui fait de sa personne le rempart physique que sa ville ne possède pas ; la Spartiate par excellence, c'est celle qui fait de sa personne le rempart éthique qui formule l'eunomia, la constitue et la renforce. Autrement 
dit, dans cette liste de voix de femmes spartiates, c'est une voix particulière qui se fait entendre, la voix, non de la cité, ni même de ses institutions, mais de l'ordre qui les régit.

\section{La voix de l'ordre}

Ordre et voix sont intimement liées à Sparte, et se trouvent à la source même de l'organisation politique : il suffit de prendre les différentes anecdotes qui circulent autour de Lycurgue, législateur originaire et auteur de l'ordre spartiate, pour s'en convaincre. Chez Hérodote, c'est à Delphes qu'ordre et voix s'unissent autour de ce personnage : à peine Lycurgue est-il entré dans le sanctuaire d'Apollon que la voix de la Pythie (une autre voix dont le genre est à discuter) résonne, le saluant et proclamant son hésitation à le traiter en homme ou en dieu :

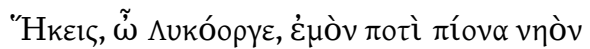

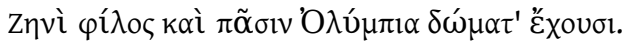

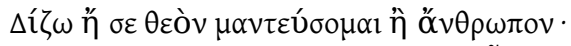

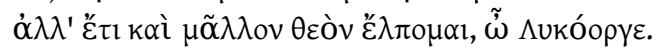

Te voici, Lycurgue, en mon temple opulent,

Toi qui es cher à Zeus, cher à tous les habitants de l'olympe.

Te proclamerai-je dieu ou mortel ? J'hésite,

Mais, Lycurgue, je te crois plutôt un dieu.

(Hérodote, I, 65)

La Pythie n'en reste d'ailleurs pas là, puisqu'elle aurait également « délivré » ( $\varphi \rho \alpha ́ \sigma \alpha l)$ l'ordre établi aujourd'hui chez les Spartiates » (

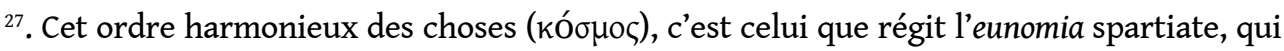
s'incarne dans un énoncé oral, forcément oral, la rhètra ${ }^{28}$. Chez Plutarque, ce n'est pas à Delphes, mais en Crète que Lycurgue trouve principalement les fondements de son organisation, en fréquentant les notables et en recueillant divers règlements, mais surtout en s'associant au musicien Thalétas, dont les chants "étaient autant d'exhortations à l'obéissance et à la concorde $»^{29}$. Ordre et voix, injonction et persuasion, parole et rhètra, voilà les termes sans cesse redoublés qui disent l'eunomia spartiate, l'harmonie de la cité et le juste rapport entre ses habitants.

Le cas spartiate est loin d'être unique et peut être inséré dans une étude sur les rapports entre loi écrite et loi orale en Grèce, dans le cadre d'une réflexion sur l'origine de la loi et la figure du législateur ${ }^{30}$. Si la rhètra spartiate a rapport avec l'ordre rythmique du musicien Thalétas, on retrouve une même association entre loi et production musicale dans les récits qui concernent les législateurs Zaleucos et Charondas, où il question en particulier d'un préambule (prooimion) qui introduisait leurs dispositions législatives, et de la possibilité même de chanter la loi : d'après Hermippos ${ }^{31}$, «à Athènes, même les lois de Charondas étaient chantées en accompagnement du vin » et d'après Poseidonios ${ }^{32}$, les Mazacéniens, qui avaient recours aux lois de Charondas, disposaient en outre d'un " chantre des lois qui a pour tâche de leur expliquer les lois, comme les jurisconsultes à Rome ». C'est dans ce cadre que l'on pourrait réenvisager les rapports entre Tyrtée et l'ordre spartiate, tant au moment de la production de ses élégies que dans les siècles qui suivirent.

Il me semble qu'il existe cependant bien une spécificité spartiate, au moins dans l'idéologie qui a été construite à son sujet, qui est la production d'un énoncé injonctif devenu exemplaire, dont les termes sont déjà proposés de façon dispersés par Hérodote et qui se réunissent, dans la formulation apophtegmatique, chez Plutarque. 
Indépendamment des formulations du mythe spartiate par Platon ou Aristote ${ }^{33}$ et de leur

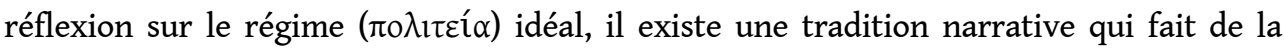
parole l'agent suprême qui établit l'ordre et qui construit une série de couples associant masculin et féminin, sans réduire cependant les membres du couple à leur genre. À la Pythie s'adressant à Lycurgue succède Gorgô admonestant son père ou les Spartiates, et plus généralement toutes les mères laconiennes qui incitent leur fils à faire don d'euxmêmes pour leur patrie, incarnant chacune à sa façon la rhètra, la voix suprême de Sparte, qui n'est nulle part et partout, l'injonction définitive de l'eunomia.

Contre cette parole spartiate, il existe d'ailleurs une autre parole chez Hérodote, celle de Solon, dans le même livre que celui où se construit la figure de Lycurgue. C'est une parole prononcée entre hommes, le sage athénien et le roi Crésus, une parole non injonctive, faite pour persuader, qui s'appuie sur des sentences et maximes et qui fait écho aux compositions orales de Solon, élégies que chantent les Athéniens, parallèles et non symétriques aux élégies de Tyrtée chantées par les Spartiates. Par cette parole tout aussi ordonnatrice, mais qui ne relève ni de l'apophtegme, ni de la surveillance et donc qui n'énonce peut-être pas une norme, se dit un autre rapport à la loi, à l'ordre et au monde.

\section{BIBLIOGRAPHIE}

BRULÉ P. et PIOLOT L. (2002), « La Mémoire des pierres à Sparte. Mourir au féminin : couches tragiques ou femmes hiérai? (Plutarque, Vie de Lycurgue, 27, 3) », Revue des Études grecques 115, Paris, p. 485-517.

CARTLEDGE P. (1979), Sparta and Lakonia. A Regional History, 1300-362 BC, coll. States and Cities of Ancient Greece, Londres.

CARTLEDGE P. (1981), « Spartan Wives », Classical Quarterly 31, p. 84-105, Oxford.

DELATTRE Ch. (2001), Les figures de Minos dans l'imaginaire antique : thalassocrate, législateur, juge infernal, thèse de l'Université de Paris X Nanterre.

DELG, Chantraine P., avec la collab. de Taillardat J., Masson O. et Perpillou J.-L., sous la dir. de

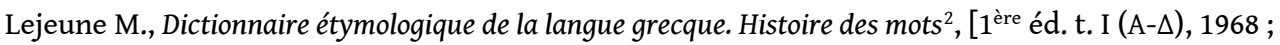

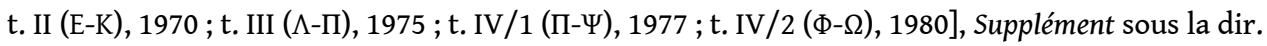
de Blanc A., de Lamberterie C., Perpillou J.-L., Paris.

DEWALD C. (1981), « Women and Culture in Herodotus'History », dans Foley H. P. éd., Reflections of Women in Antiquity, New York-Tokyo.

CORCELLA A. (1985), « Erodoto VII, 239 : una 'interpolazione d'autore' », Annali della Scuola Normale di Pisa, série III, XV, 2.

FRASER P. et MATTHEWS E. éd. (1987-2005), A Lexicon of Greek Personal Names (The Aegean Islands, Cyprus, Cyrenaica), t. I, Oxford.

LÉVY E. (2003), Sparte. Histoire politique et sociale jusqu'à la conquête romaine, Paris.

NACHSTÄDT W. éd. (1935), Plutarchi Moralia, t. II, Leipzig. 
OLLIER F. (1933-1943), Le mirage spartiate. Étude sur l'idéalisation de Sparte dans l'antiquité grecque. t. I : de l'origine jusqu'aux cyniques. t. II : du début de l'école cynique jusqu'à la fin de la cité, Paris.

PARADISO A. (2003), « Gorgô, la Spartiate », dans Loraux N., La Grèce au féminin, trad. Monsacré H.,

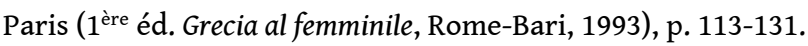

POMEROY S. (2002), Spartan Women, Oxford.

ROMERO GONZÁLEZ D. (2008), « El prototipo de mujer espartana en Plutarco », dans Nikolaidis A. G. éd., The Unity of Plutarch's Work. 'Moralia' Thems in the 'Lives', Features of the 'Lives' in the 'Moralia', Berlin-New York, p. 679-687.

STADTER P. A. (2008), « Notes and Anecdotes : Observations on Cross-Genre Apophthegmata », dans Nikolaidis A. G. éd., The Unity of Plutarch's Work. 'Moralia' Thems in the 'Lives', Features of the 'Lives' in the 'Moralia', Berlin-New York, p. 53-66.

TIGERSTEDT E. N. (1965-1978), The Legend of Sparta in Classical Antiquity, Stockholm.

VAN DER STOCKT L. (1999), «Three Aristotles equal but one Plato : on a cluster of quotations in Plutarch », dans Pérez Jiménez A., García López J. et Aguilar R. éd., Plutarco, Platón y Aristóteles. Congreso internacional de la I.P.S. Madrid-Cuenca (4-7 de mayo de 1999), Madrid, p. 127-140.

VAN DER STOCKT L. (2004), «Plutarch in Plutarch : the problem of the Hypomnemata », dans Gallo I. éd., La Biblioteca di Plutarco. Convegno plutarcheo. Pavia, 13-15 June 2002, Naples, M. D’Auria, p. 331-340.

VAN MEIRVENNE B. (1999), « Puzzling over Plutarch. Traces of a Plutarchean Plato-study concerning Lg. 729A-C in Adulat. 32 (Mor. 71B), Coniug. Praec. 46- 47 (Mor. 144F) and Aet. Rom. 33 ( Mor. 272C) ", dans Montes Cala J. G., Sánchez Ortiz de Landaluce M. et Gallé Cejudo R. J. éd., Plutarco, Dioniso y el vino. Actas del VI simposio español sobre Plutarco (Cádiz, 14-16.5.1998), Madrid, p. 527-540.

\section{NOTES}

1. Pour l'alliance que l'entreprise plutarquéenne réalise en général entre anecdote historique et èthos, cf. Plutarque, Alexandre, $1,2$.

2. Cf. par exemple Polybe, I, 2 ; Denys d'Halicarnasse, Antiquités romaines, I, 2, 2-3, 3.

3. Les formulations figées de ce mythe spartiate, institutions particulières et dénominations locales - cryptie, sissyties, etc. - ont été particulièrement mises en valeur par François olLIER (1933-1943) et Eugène N. TIGERSTED (1965-1978). Pour une réévaluation historique de ces éléments, cf. CARTLEDGE 1979 et LÉVY 2003.

4. Cf. l'introduction de Fr. FUHRMANN à l'édition dans la CUF (1988, p. 6-9 et p. 131-135). L'étude de cette question a été renouvelée par l'analyse des clusters (énoncés centrés sur une anecdote ou un thème que l'on trouve à plusieurs reprises dans l'œuvre de Plutarque et qui remonteraient à des hypomnemata ou notes préliminaires) menée par L. VAN DER STOCKT (1999 et 2004) et B. VAN MEIRVENNE, et appliquée aux recueils d'apopthegmes plutarquéens par Ph. STADTER et M. BECK (voir en dernier lieu STADTER 2008, p. 53-54).

5. Il y a d'ailleurs quelques reprises entre les deux premières séries et la dernière: un apophtegme de Gorgô $\left(\mathrm{n}^{\circ} 5,240 \mathrm{E}\right)$ a déjà été inséré sous son nom dans les énoncés attribués à Lycurgue $\left(n^{\circ} 13,227 E\right)$. Un autre $\left(n^{\circ} 2,240 \mathrm{D}\right)$ est attribué à Archidamos II $\left(n^{\circ} 6,218 \mathrm{C}\right)$. À l'inverse, le sixième apophtegme qui est enregistré sous son nom est en fait un énoncé prononcé par son époux Léonidas et se trouve donc à sa place dans les apophtegmes attribués à ce dernier $\left(\mathrm{n}^{\circ} 2\right.$, 
225A). Certains apophtegmes sont repris en dehors de la collection : C. Baroin me signale par exemple que l'apophtegme de Laconienne $\mathrm{n}^{\circ} 13,241 \mathrm{E}$ est prononcé par Alexandre à l'intention de son père Philippe dans un traité de Plutarque, Sur la fortune d'Alexandre, I, 9, 331B, et par la mère de Sp. Carvilius à l'intention de son fils chez Cicéron, De oratore, II, 249.

6. Seules exceptions, Gorgô, dont deux apopthegmes concernent son père ( $\left.n^{\circ} 1-2,240 \mathrm{D}\right)$, et deux autres, un étranger en visite ou résidence $\left(\mathrm{n}^{\circ} 3-4,240 \mathrm{E}\right)$; les derniers apophtegmes de Laconiennes anonymes ne concernent pas directement leur statut de mère, mais plutôt celui d'épouse ( $\left.n^{\circ} 23-25,242 B-C\right)$. Les derniers apophtegmes ( $\left.n^{\circ} 26-30,242 C-D\right)$ concernent des valeurs

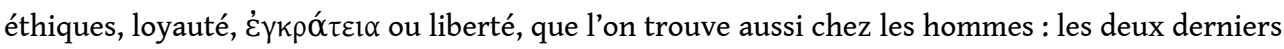
sont même attribués à des Spartiates anonymes ( $\left.n^{\circ} 29=n^{\circ} 39,234 \mathrm{C} ; \mathrm{n}^{\circ} 30=\mathrm{n}^{\circ} 37-38,234 \mathrm{~B}-\mathrm{C}\right)$.

7. Stobée, III, 7, 30, qui fait de Gorgô, épouse de Léonidas, l'auteur de ce mot; cf. Sextus Empiricus, Hypotyposes, III, 216 ; Valère-Maxime, II, 7, ext. 2 ; Sénèque, Suasoriae, II, 8.

8. Cf. Tyrtée, 8 Diehl, particulièrement v. 11-13, qui font l'éloge du corps à corps et v. 29-34, qui font de ce corps à corps un "armes à armes ", épée, bouclier et panache s'entrelaçant à ceux de l'adversaire.

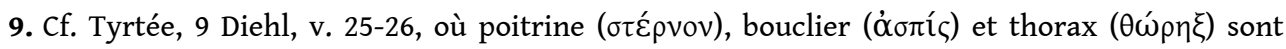
pareillement transpercés par les coups de l'adversaire.

10. L'apophtegme anonyme $n^{\circ} 34,239 B$, cite d'ailleurs ces vers et rapporte qu'Archiloque fut chassé de Sparte pour les avoir composés.

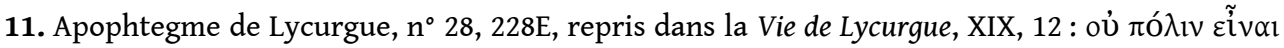

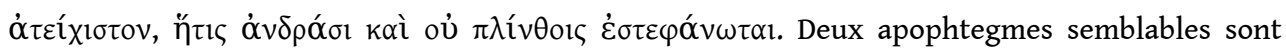
attribués à Agésilas, $\mathrm{n}^{\circ} 29$ et 30,210E.

12. Un apophtegme de Lycurgue $\left(n^{\circ} 12,227 \mathrm{D}\right)$ justifie l'éducation physique des filles par la nécessité d'engendrer des enfants solides. Un autre $\left(n^{\circ} 16,228 \mathrm{~A}\right)$ fixe pour les deux sexes un certain âge pour le mariage, pour les mêmes raisons.

13. Sarah POMEROY (2002, p. VIII) : "It must be emphasized that often the primary sources do not distinguish between prescriptive and descriptive writing; and pictorial representations may also be idealistic or fantastic rather than realistic. (...) I must, however, confess that my tendency is to grant more credence to the primary sources than some contemporary hypercritical Spartanologists are wont to do, and to understand that they generally reflect an actual historical situation rather than a utopian fiction " (je souligne). La distinction que S. Pomeroy fait entre sources primaires et secondaires, entre "réalité historique» et "fiction utopique", me semble insuffisante pour le traitement des apophtegmes. Le chercheur ayant horreur du vide, il peut supposer que ces apophtegmes ne se fondent pas seulement sur un fantasme, mais reconstituer le noyau historique qui serait la Sparte véritable à partir de ces mêmes apophtegmes est une opération qui aboutit à la construction d'un échafaudage aussi hypothétique qu'invérifiable.

14. POMERoY (2002, p. 60), à propos de l'apophtegme de Gorgô, $\mathrm{n}^{\circ} 5,240 \mathrm{E}$. Toute l'analyse des apophtegmes de femmes laconiennes vise dans cet ouvrage à reconstruire une "Ideology of Motherhood» (titre du chapitre, p. 57) qui serait celle de la Sparte d'époque classique. De façon plus distanciée, à la suite de Paul CARTLEDGE (1981), Dámaris ROMERO GONZÁLEZ (2008) reconstruit à partir de Plutarque un « prototype de la femme spartiate » dont elle précise qu'il s'agit seulement d'une « image » qui n'est pas le reflet direct de la réalité historique.

15. Dámaris ROMERo GONZÁLEZ (2008, p. 687) souligne l'existence de l'écart qui se creuse entre la norme définie par les apophtegmes plutarquéens et les anecdotes présentées dans les Vies, où des femmes spartiates s'arrangent des règles d'austérité et d'obéissance à l'égard des hommes. Elle fait cependant de cet écart le résultat d'un projet narratif - exemplarité du prototype féminin construit dans les Moralia, prise en compte de la réalité historique dans les Vies - alors que la distinction est opératoire déjà dans les Apophtegmes laconiens.

16. Cf. apophtegme de Lycurgue, $n^{\circ} 25,228 \mathrm{D}$ : les Spartiates ne doivent pas s'attaquer aux remparts d'une ville, mais seulement à ses soldats, afin que ceux qui sont «meilleurs » 
( $\dot{\alpha} \mu \varepsilon i ́ v o v \varepsilon \varsigma)$ ne courent pas le risque de mourir de la main «d'une femme, d'un enfant ou d'un individu du même genre » (ن் terme ớ $v \theta \rho \omega \pi$ o dans l'énumération montre bien que la différence se fait par le statut, non par le genre ou l'âge, qui sont seulement des critères constitutifs parmi d'autres.

17. Une correction de K. Latte au texte de la Vie de Lycurgue, 27, 2, faite par K. Ziegler dans une édition de 1926 chez Teubner à la suite d'une remarque inédite de K. Latte, a longtemps fait croire qu'une forme d'égalité existait dans le traitement funéraire de certains hommes et de

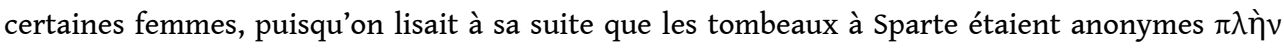

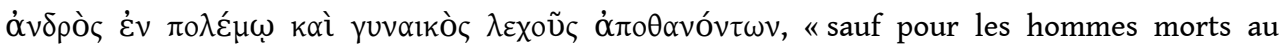
combat et les femmes mortes en couches» (cf. POMEROY 2002, p. 52 et n. 3 p. 52, parmi d'autres). Une mise au point récente de Pierre BRULÉ et Laurent PIOLOT (2002) incite à abandonner cette

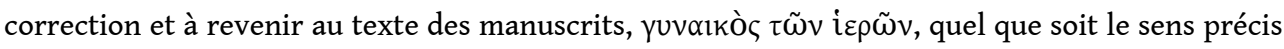
que l'on donne à ces ípaí. D'ailleurs un passage parallèle des Apophtegmes laconiens, 238D («Institutions », $n^{\circ} 18$ ), qui évoque les usages funéraires imposés par la législation de Lycurgue, ne mentionne que les hommes, et non les femmes.

18. Ce point est pratiquement absent de la bibliographie, qui s'obstine à parler de «femmes spartiates "; seul Paul CARTLEDGE (1981) établit une utile distinction à la fois entre les catégories sociales (femme pauvre, femme riche, etc.) et entre les fonctions sociales (mère, épouse, etc.).

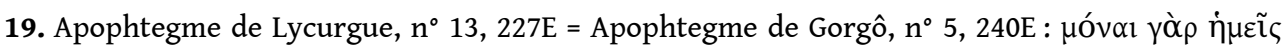

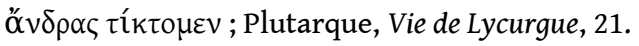

20. Apophtegme anonyme, $n^{\circ} 10-11,241 \mathrm{D}-\mathrm{E}$.

21. Anecdote de Damatria, qui tua son propre fils, au témoignage d'une épigramme funéraire : « Pour avoir enfreint les lois, Damatrios fut tué par sa mère : c'est une Lacédémonienne, c'est un

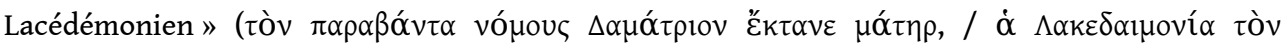
$\Lambda \alpha \kappa \varepsilon \delta \alpha$ uóviov).

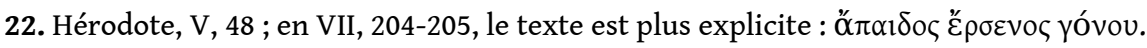

23. Hérodote, V, 48-51; Plutarque, Apopht. Lac., 240D-E. L'épisode redouble celui où Cléomène résiste de lui-même à Maiandros de Samos (Hérodote, III, 148).

24. Hérodote, VII, 239. Cf. DEWALD 1981, CORCELLA 1985 et PARADISO 2003.

25. Cf. FRASER et MATTHEWS, 1987-2005.

26. Hérodote emploie le verbe $\mu \eta \chi \alpha v \tilde{\alpha} \sigma \theta \alpha$ t pour qualifier l'action de Démarate sur la tablette.

27. Ibid. Hérodote prend ses distances avec cette anecdote : il rapporte en effet la version des Spartiates eux-mêmes, pour qui Lycurgue aurait ramené de Crète son idée de l'ordre juste.

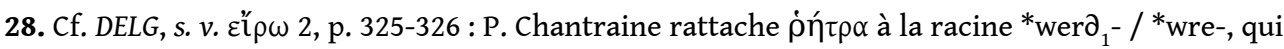
exprime l'idée de «formuler », « dire la formule ».

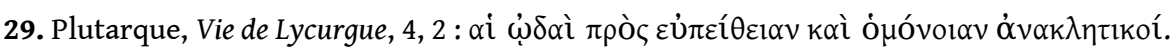

30. Je renvoie ici au chapitre de ma thèse « La figure du législateur » (DELATTRE 2001).

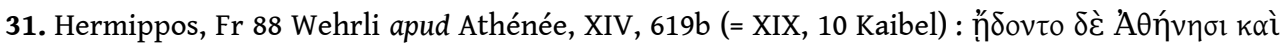
oi X $\alpha \rho \omega ́ v \delta$ ov vónoı $\pi \alpha \rho^{\prime}$ oĩvov.

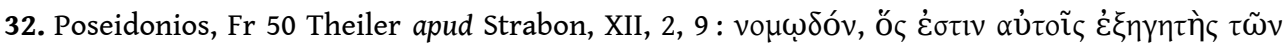

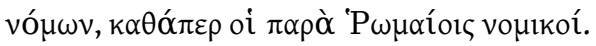

33. D'après Plutarque, Vie de Lycurgue, 14, Aristote critiquait la liberté des femmes spartiates, une façon de réinterpréter le modèle de la voix laconienne en transformant l'injonction qui établit l'ordre en facteur de désordre. 


\section{RÉSUMÉS}

Le recueil plutarquéen des Apophtegmes laconiens réunit plusieurs séries de formules frappantes, mises au compte de Spartiates. Le catalogue s'organise suivant plusieurs critères, en particulier celui qui oppose individus célèbres et anonymes, mais aussi celui qui distingue hommes et femmes. Mais les thèmes abordés dans les apophtegmes permettent de construire un éthos qui ne repose pas exactement sur le critère du genre : la parole des femmes spartiates qui résonne dans le volume fait de celles-ci des mères, et celle de leurs enfants mâles, exclusivement des soldats. La parole elle-même est spécifique : comme le montre l'exemple de Gorgô, elle est énoncée comme injonction, garantit le maintien de l'eunomia spartiate et redouble une autre parole, celle de la rhètra. Cette parole propre à Sparte s'oppose à celle de Solon à Athènes, où la maxime et la loi se disent entre hommes, excluant les mères et instaurant une isègorie absente de Sparte.

In the Laconian Apophthegms, Plutarch gathers several series of striking sayings uttered by Spartans. The catalog is organized according to several criteria, particularly that between famous and anonymous people, but also that which distinguishes men and women. But the themes of the sayings build an ethos that is not exactly based on the criterion of gender: the words that resonate in the volume define women first of all as mothers, and at the same time define their children exclusively as male soldiers. The form of speech itself is specific: as the example of Gorgo shows, it is stated as an order, ensuring the maintenance of Spartan eunomia and it follows another speech, that of rhetra. The speech, peculiar to Sparta, differs radically from Solon's speech in Athens, where the maxim and the law are discussed among men, excluding mothers and introducing an isegoria absent from Sparta.

\section{INDEX}

Keywords : apophthegm, civic order, eunomia rhetra, gender, mother, Plutarch, Sparta

Mots-clés : apophtegme, eunomia rhètra, genre, mère, ordre civique, Plutarque, Sparte

\section{AUTEUR}

\section{CHARLES DELATTRE}

Université de Paris Ouest Nanterre La Défense, THEMAM (Textes, Histoires et Monuments de l'Antiquité au Moyen Âge) - UMR 7041 ArScAn (Archéologie et Sciences de l'Antiquité) 